\title{
REHABILITACIÓN BASADA EN LA COMUNIDAD FRENTE A LA REALIDAD CHILENA ${ }^{1}$
}

\author{
COMMUNITY-BASED REHABILITATION FACING THE CHILEAN REALITY
}

\section{María Paz Ossandón P.²}

\begin{abstract}
Resumen
El desarrollo histórico del conocimiento y el acercamiento de la salud a los distintos grupos sociales han promovido el paso desde un enfoque biomédico a uno biopsicosocial. Bajo esta mirada se gestó hace 32 años la Rehabilitación Basada en la Comunidad $(R B C)$ como una estrategia de inclusión y promoción de los derechos de las personas con discapacidad.

La implementación de la nueva forma de rehabilitación en Chile ha resultado ser tardía. El escenario actual del país impone múltiples obstáculos para permitir el desarrollo de este modelo. Dadas las características de la sociedad chilena y de sus profesionales, ¿es posible alcanzar las metas y objetivos propuestos para la RBC?

En el presente ensayo se describe la realidad chilena y la factibilidad actual de implementar un programa de RBC, siguiendo las guías propuestas por la OMS (2012) y las orientaciones dictadas por el Ministerio de Salud (2010). En éste se logran identificar los principales obstáculos presentes en el país, los que se relacionan con la resistencia social, política y profesional hacia la RBC.
\end{abstract}

\section{Palabras Claves:}

Rehabilitación basada en la comunidad, personas con discapacidad, integración social, modelo biopsicosocial.

\begin{abstract}
The historical development of knowledge and the health approach to different social groups have promoted the transition from a biomedical approach into a biopsychosocial one. From this perspective, 32 years ago the Community-based Rehabilitation (CBR) was conceived, as a strategy of inclusion and promotion of the rights of the people with disabilities.

The implementation of the new way of rehabilitation in Chile has been late. The current stage of the country imposes multiple obstacles to the development of this model. Considering the Chilean society characteristics and it professionals, Is it possible to reach the goals and aims proposed for the CBR?

In this essay is described the Chilean reality and the current feasibility of implementing a CBR program, following the guidelines proposed by the WHO (2012) and the orientations dictated by the Health Ministry (2010). In this essay is possible to identify the main obstacles in Chile related to the social, political and professional resistance to the CBR.
\end{abstract}

\section{Key words:}

Community-based rehabilitation, people with disabilities, social integration, biopsychosocial model.

1 Trabajo correspondiente al estudio y análisis realizado por la autora para optar al título de Terapeuta Ocupacional en la Universidad de Chile, aprobado con distinción máxima en el año 2013.

2 Terapeuta Ocupacional, Licenciada en Ciencias de la Ocupación Humana, Universidad de Chile. Contacto: mp.ossandon@gmail.com 


\section{INTRODUCCIÓN}

La Organización Internacional del Trabajo (OIT), la Organización de las Naciones Unidas para la Educación, la Ciencia y la Cultura (UNESCO) y la Organización Mundial de la Salud (OMS) en su posición conjunta en el año 2004, plantean la Rehabilitación Basada en la Comunidad (RBC) como una "estrategia de desarrollo comunitario para la rehabilitación, la igualdad de oportunidades y la integración social de todas las personas con discapacidad". Ésta surge en respuesta a la exclusión y a la dificultad en el acceso a la salud, principalmente en los servicios de rehabilitación, que vivían las personas con discapacidad en todo el mundo.

La adquisición de las nuevas estrategias de rehabilitación e intervención en Chile ha resultado ser tardía. Recién en el año 2003 el enfoque de RBC comenzó a ser implementado como respuesta a la inequidad en el acceso a la salud. Para esto, de acuerdo a lo descrito por el Ministerio de Salud (MINSAL) en 2010, se incorporaron las bases de la RBC desde la política pública tomando como eje las propuestas entregadas por la Reforma de Salud (2000), la epidemiología social y epidemiología de la discapacidad, con el objetivo de contar con una red de establecimientos de salud y redes sociales que brinden una mayor y mejor cobertura para las comunidades. Para lograr un cambio en la forma de intervenir en salud en Chile, el MINSAL propone un nuevo equipo de trabajo que se encargue de asesorar, promover y guiar a las personas con discapacidad y sus familias para que consigan integrarse satisfactoriamente en su comunidad.

Según las pautas nacionales e internacionales de implementación de la $\mathrm{RBC}^{3}$, el Terapeuta Ocupacional es parte esencial del equipo de in-

3 A nivel nacional propuesto por "Orientaciones Metodológicas para el Desarrollo de las Estrategias de Rehabilitación en APS 2010 -2014" (MINSAL, 2010) y a nivel internacional por: "Rehabilitación Basada en la Comunidad: Guías para la RBC" (OMS, 2012) tervención, por lo que el proceso de desarrollo de la nueva forma de rehabilitación en Chile resulta transcendental para la profesión. Asimismo, ha permitido a los nuevos terapeutas ocupacionales ampliar su campo laboral e integrar los nuevos conceptos dictados por la RBC, durante su formación y en su posterior desempeño profesional. Considerando que las nuevas tareas de los equipos de rehabilitación se relacionan con el acompañamiento y facilitación de oportunidades para las personas con discapacidad y su entorno, es fundamental que su perfil se vincule con los aspectos comunitarios.

En este proceso de implementación de una nueva visión de la Salud en Chile han surgido dificultades y barreras que entorpecen el desarroIlo de la RBC. De igual forma, existe un distanciamiento del proceso entre el país y las pautas planteadas a nivel internacional. Es por esto que el análisis de la factibilidad actual de aplicar las estrategias propuestas por la OMS en 2012 resulta de gradual importancia. Más aún considerando las características socio-económicas de la población nacional, los recursos humanos disponibles y las actuales políticas impulsadas por el Gobierno, en especial por el MINSAL.

\section{DesarRollo temÁtico}

\section{RBC y los cambios en la forma de intervenir en salud}

De acuerdo a lo planteado por la Organización Panamericana de Salud (OPS) en 2011, la RBC se enmarca dentro del modelo biopsicosocial, buscando replantear las intervenciones y visiones tanto de los individuos como de las atenciones y prestaciones de salud propuestas por el modelo biomédico. Según Oyarzún (2008), en el antiguo modelo la discapacidad es vista como un problema propio e inherente al individuo, pues es él quien presenta deficiencias que dificultan su desempeño y participación. Además las intervenciones y prestaciones de salud se orientan a solucionar las dificultades de 
manera unidireccional, es decir, la persona no participa en su proceso de tratamiento sino que sólo es un receptor. Por lo anterior se podría considerar la visión del modelo biomédico como reduccionista, ya que aborda las enfermedades desde conceptos biológicos, ignorando aquellos aspectos sociales, ambientales o psicológicos del individuo. Asimismo, debido al asistencialismo de sus prácticas, podría carecer de dialéctica, pasando por alto la responsabilidad y compromiso de las personas con su salud. Por su parte, Oyarzún (2008) plantea que el modelo biopsicosocial concibe al individuo de manera holística, considerando tanto los aspectos personales como ambientales que podrían interferir en el proceso de salud-enfermedad. Desde este punto de vista, la discapacidad no es entendida como una característica del individuo, sino que corresponde a la interacción de las dificultades personales y las barreras físicas y sociales del entorno. Además deja atrás la lógica asistencialista, entregando a las personas y sus comunidades la responsabilidad de ser actores y productores de su propia salud.

Bajo las consideraciones del propio modelo biopsicosocial y basada en lo propuesto por las Guías para la RBC (OMS, 2012) la estrategia de RBC implica cambios en la forma de intervenir en salud, por consiguiente en las acciones emprendidas por los profesionales encargados de estas tareas.

Para comprender de mejor manera lo descrito previamente, a continuación se detallan algunos de los importantes cambios que implica esta transición:

- Una de las principales características de la RBC es el empoderamiento de los individuos y de sus comunidades, quienes bajo la nueva visión están encargados de trabajar en pro de su salud, por lo que el mejoramiento de las condiciones de salud y calidad de vida de los individuos y de su entorno pasa de estar en manos de los profesionales a ser una labor de la comunidad. Para lograr éste objetivo, la idea es que exista una comunicación directa entre ambas partes, donde se entreguen estrategias y se compartan los saberes profesionales, otorgando las principales herramientas a las personas con discapacidad y su comunidad, donde los conocimientos profesionales ya no serán exclusivos ni impuestos a la comunidad. Esto permite una democratización del conocimiento, cuya base es la descentralización de las responsabilidades y de los recursos. Dicho propósito se podría realizar en base a la educación popular planteada por Freire (1970), donde los conocimientos son compartidos bajo una lógica dialéctica y constructiva, en una relación horizontal en la que exista una cooperación entre el profesional y las personas (Kolmans, s.f). Como los terapeutas ocupacionales, son parte fundamental del equipo de rehabilitación dictado por documentos nacionales e internacionales, es primordial que estos profesionales asuman los nuevos desafíos dictados por la RBC.

- Otro cambio primordial se refleja en las acciones de los equipos de salud, puesto que no se enmarcan a la asistencia e intervención propia de cada disciplina, sino que se suman funciones de asesoramiento y facilitación de los procesos de RBC dentro de la propia comunidad.

- Por otra parte, existe una variación en el lugar donde se realizan las intervenciones en salud, pues desde la lógica biomédica, estas eran entregadas en un Hospital de manera centralizada y muchas veces excluyente, ya que impedía el acceso de todas las personas que lo necesitaban. Bajo el modelo biopsicosocial y la estrategia de RBC, las intervenciones son realizadas tanto por los profesionales como por los propios individuos en la comunidad, donde existe una red que asesora y permite la entrega de prestaciones oportunas y adecuadas para cada persona.

\section{Desarrollo Internacional de la RBC}

Para comprender las bases y cómo ha surgido la RBC de manera internacional, es importante co- 
nocer los hitos históricos que ponen de manifiesto su desarrollo. Los comienzos de la nueva estrategia se remontan a los años setenta, caracterizado por un panorama económico y político crítico en el mundo, donde las democracias eran poco estables, la exclusión social en todos los sectores era abismante, las personas con discapacidad no eran consideradas dentro de las políticas públicas y donde la salud poseía una orientación centralista y deficiente (Alcalde, 1981). Bajo este panorama, en 1978 la OMS publica la "Declaración de Alma Ata" que busca ser la base para la construcción de un nuevo sistema de salud inclusivo y cercano a las comunidades. En ella se insta a los gobiernos a formular políticas nacionales que apunten a desarrollar e implementar la Atención Primaria en Salud (APS) como prioridad en las intervenciones, planteándola como el método para promover la justicia social, ya que facilita el acceso a la salud de las poblaciones y el acercamiento a la comunidad.

Las condiciones socio económicas internacionales a finales de la década de los setenta impidieron el progreso hacia una salud inclusiva, debido a la recesión mundial y al aumento creciente de la pobreza. Como una respuesta a las dificultades, en 1981 la OMS publicó un manual de RBC el que describe claramente la estrategia en términos operativos, destacando el valor de las acciones en la comunidad, principalmente por el costo de efectividad de sus intervenciones. En éste manual se incluyen guías donde se exponen estrategias con el objetivo de acercar las prestaciones de rehabilitación a la comunidad.

Con la base entregada por la OMS en 1981 y bajo un clima mundial donde las personas con discapacidad comenzaban a ser consideradas por las instituciones y gobiernos, en 1993 la Organización de las Naciones Unidas (ONU) publica las "Normas Uniformes sobre la igualdad de oportunidades para las personas con discapacidad", donde se pone de manifiesto el compromiso moral y político de actuar para igualar las oportunidades de las personas con discapacidad. Mediante 22 normas se establecen importantes principios de responsabilidad, acción y cooperación dirigidos al mejoramiento de la calidad de vida y la plena participación de las personas con discapacidad en igualdad de condiciones. Asimismo, apunta a medidas de sensibilización en la comunidad, acceso a distintos sectores como salud, educación y empleo en igualdad de oportunidades.

Posteriormente en 1994, la OIT, la UNESCO y la OMS elaboraron un "Documento de Posición Conjunta" sobre la RBC para fomentar un enfoque común del desarrollo de los programas de RBC y así alcanzar una salud igualitaria y accesible. En él se desarrolla conceptualmente la estrategia, donde se le otorga especial importancia al rol de la comunidad dentro de las acciones e intervenciones. Asimismo se promueve el trabajo intersectorial para lograr la inclusión de las personas con discapacidad.

Bajo un panorama donde la RBC comenzaba a tomar fuerza y ser implementado por diversos países, considerando la evolución de las prácticas y los cambios conceptuales en torno a la discapacidad, en 2002 la OMS edita los manuales publicados previamente en 1981, donde a través de una variedad de cuadernillos se entregan estrategias de intervención y conceptualización de las principales dificultades de integración social de las personas con discapacidad.

Considerando la evolución de la RBC y tras 25 años de su implementación, en 2003 se lleva a cabo una "Consulta Internacional para Revisar la RBC" organizada por la OMS. En ella se reconoce que la estrategia de $\mathrm{RBC}$ ha evolucionado ampliando sus prácticas y conceptos e incluyendo como foco principal los derechos humanos de las personas con discapacidad en sus comunidades. Dentro de esta consulta se postularon un número de recomendaciones que son expuestas posteriormente en la $2^{\circ}$ posición conjunta de la OIT, UNESCO y OMS en 2004 donde se describe y respalda la RBC como una "estrategia dentro del desarrollo comunitario general para la rehabilitación, la equiparación de oportunidades, la reducción de la pobreza y la inclusión social de las personas con discapaci- 
dad", poniendo especial énfasis en los derechos humanos y el fortalecimiento de la comunidad, haciendo un llamado a los distintos gobiernos a implementar acciones intersectoriales para combatir la pobreza.

Posteriormente en 2006 la ONU, basándose en su publicación de 1993, realiza la "Convención Internacional sobre los Derechos de las personas con discapacidad". Con ella no se pretende crear nuevos derechos exclusivos para este grupo, sino que los derechos humanos compartidos por todos los individuos incluyan y sean accesibles para las personas con discapacidad. Bajo esta convención se abordan temas de accesibilidad, salud, educación y empleo, donde se esquematizan medidas para que los estados partes aseguren el cumplimiento de los derechos de las personas con discapacidad.

Finalmente en 2012 la OMS vuelve a publicar un manual llamado "Guías para la RBC" donde se actualizan las prácticas. En ella ya no se incluyen exclusivamente recomendaciones para la rehabilitación y trabajo en salud, sino que mediante una matriz se promueve la inclusión de las personas con discapacidad en todos los sectores del desarrollo. Asimismo se le entrega especial importancia al fortalecimiento de las personas con discapacidad, sus organizaciones y la comunidad en general para exigir el cumplimiento y respeto de sus derechos.

\section{Desarrollo Nacional de la RBC}

Para contextualizar el desarrollo de la RBC a la realidad chilena, es importante conocer este proceso en el país. Se puede considerar que el desarrollo de la RBC en Chile es tardío en relación al desarrollo internacional, pues una de las primeras acciones se remontan recién a 1994, donde se proclama la Ley 19.284 de la integración de las personas con discapacidad, guiada por las Normas Uniformes publicadas un año antes por la ONU. Con ésta ley se pretende tomar acciones a favor de la igualdad de oportunidades para las personas con discapacidad, considerando aspectos de salud, educación, trabajo y acceso en general a la sociedad. De esta manera, se comienzan a abordar los temas de discapacidad en Chile, considerando a uno de los grupos que hasta esa fecha había sido ignorado.

Frente al vasto desarrollo internacional de la RBC y a 25 años de su existencia a nivel mundial, en 2003 el MINSAL impulsa el desarrollo e implementación de proyectos y programas de RBC en centros de APS con financiamiento de la cooperación japonesa. Así se comienza a incorporar la estrategia desde la política pública (FONADIS, 2006).

Posteriormente en 2004, el MINSAL desarrolló una serie de compromisos con la idea de lograr la "igualdad de oportunidades y la promoción de los derechos humanos de las personas con discapacidad" (FONADIS, 2006). Los principales compromisos se relacionan con el desarrollo de una Política Nacional de Rehabilitación y Discapacidad junto con el desarrollo de un sistema de rehabilitación física y sensorial basado en la comunidad, con respaldo de manuales y guías clínicas para reforzar las acciones sanitarias. Por otra parte, en ese mismo año, se publica el primer y único estudio nacional de la discapacidad ENDISC-CIF (FONADIS, 2004), donde se da a conocer la real estadística de las personas con discapacidad en Chile. A través de ello se reconoce que hacia el año 2004 existían en el país un número superior a 2 millones de personas con discapacidad, quienes representan el $12,9 \%$ de la población total. Por otra parte, el estudio entrega información valiosa y alarmante en relación al acceso y atención de salud de este grupo, donde se indica que sólo el $6,5 \%$ de la población con discapacidad tiene acceso a rehabilitación, volviéndose imperioso el emprendimiento de medidas que apuntaran a romper con la exclusión existente para este grupo.

En 2007 el MINSAL desarrolla un programa de rehabilitación integral en la red de salud donde se estructura una lógica que apunta a adoptar la estrategia de RBC en la APS en el mediano plazo. 
Esta se enfoca en atender la discapacidad física considerando un acercamiento de las intervenciones de salud a la comunidad. Asimismo se describen las acciones a implementar por los nuevos Centros Comunitarios de Rehabilitación (CCR), que representan un proyecto en pleno desarrollo y que es dentro de este año donde se comienzan a implementar de manera masiva. Los CCR son entendidos como una "instancia de participación comunitaria que aplican la estrategia de RBC, para acoger a la comunidad y en particular a las personas con discapacidad y su familia, articulando acciones y los recursos de la comunidad para detectar y dar soluciones a sus propias necesidades de acuerdo a su realidad local" (INRPAC, 2010). Estos centros son una de las medidas pensadas desde el gobierno para implementar la RBC en Chile.

Con el avance y desarrollo de la RBC en el país y considerando la posible implementación de la nueva estrategia dentro de los CCR, en 2009 el MINSAL actualiza la información publicada en 2007, entregando orientaciones metodológicas para el trabajo de rehabilitación integral en el país. En él se muestra el modelo al cual se pretende llegar en el corto y mediano plazo. Se entregan elementos conceptuales y pasos metodológicos para adoptar la RBC como estrategia. Así, el MINSAL establece que algunos CCR comenzarán con la atención de problemas de salud física hasta finalmente incorporar el modelo de RBC completo, en el corto y mediano plazo.

Basado en su publicación en 2007 y su actualización en 2009, es finalmente en 2010 donde el MINSAL publica las "Orientaciones Metodológicas para el Desarrollo de las Estrategias de Rehabilitación en APS", que se consideran como una síntesis y avance de los trabajos anteriores. Estas orientaciones pretenden entregar elementos conceptuales para incorporar la estrategia de RBC en el desarrollo de la red pública de salud. EI MINSAL plantea como horizonte el año 2014 para dar cumplimiento a la implementación de los programas de RBC, ejecutados principalmen- te por los CCR. En 2010, también, se dicta la Ley 20.422 que establece normas sobre igualdad de oportunidades e inclusión social de personas con discapacidad. En ella se exponen "estrategias que apuntan a la disminución de discriminación por razón de discapacidad y la adopción de medidas de acción positiva orientadas a evitar o compensar las desventajas de una persona con discapacidad para participar plenamente en la vida política, educacional, laboral, económica, cultural y social" (Ministerio de Planificación del Gobierno de Chile, 2010). Asimismo la ley establece que la prevención y la rehabilitación constituyen una obligación del Estado y un derecho y un deber de las personas con discapacidad, de su familia y de la sociedad en su conjunto.

A pesar de las medidas implementadas por los distintos gobiernos y las estrategias que apuntan a posicionar la RBC como método de rehabilitación en el país, la realidad indica que las políticas no se condicen estrictamente con lo que ocurre realmente en los centros de salud en Chile. Además, las orientaciones apuntan el cumplimiento de objetivos planteando como límite el año 2014, y estando a un año de la fecha, las intervenciones si bien se han acercado a la comunidad, aún falta mucho para que tengan una base comunitaria.

\section{Diferencias entre documentos nacionales e internacionales vinculados a la RBC}

Si se pretende implementar un programa de RBC en Chile, parece adecuado considerar un análisis comparativo de las últimas publicaciones nacionales e internacionales con la finalidad de reconocer deficiencias y posibles potencialidades de la realidad del país. Dentro de este análisis se incluyen las publicaciones de la OMS en 2012 y del MINSAL en 2010, en las que se reconocen ideas símiles que abordan la inclusión de las personas con discapacidad. Sin embargo, existen notables diferencias en sus formas de concebir la RBC y la comunidad. 
Tabla 1: Análisis comparativo de documentos de la OMS y MINSAL

\begin{tabular}{|l|l|}
\hline Guías para la RBC $(\mathbf{O M S})$ & Orientaciones Metodológicas (MINSAL) \\
\hline $\begin{array}{l}\text { Entiende comunidad de acuerdo a pertenencia, } \\
\text { interrelación y cultura común }\end{array}$ & Entiende comunidad como unidad territorial \\
\hline Incluye un proceso integral de inclusión & Incluye rehabilitación física principalmente \\
\hline Proceso desde la comunidad & Proceso desde niveles centrales \\
\hline Prestaciones de salud con base comunitaria & Extensión de servicios profesionales en la comunidad \\
\hline
\end{tabular}

En relación a lo expuesto previamente en la tabla, en primer lugar la OMS en las Guías para la RBC aborda el concepto de comunidad desde una perspectiva similar a lo expuesto por Krause Jacob (2001), donde se define en base a tres puntos: la pertenencia, es decir sentirse parte o identificado con el grupo; seguido por la interrelación, que será entendido como la comunicación, interdependencia e influencia mutua entre los miembros; y finalmente el tener una cultura común, entendiendo cultura como una red de significados compartidos. Por lo que una comunidad deberá compartir, al menos en cierto grado, una visión de mundo, una interpretación de la vida cotidiana. Mientras que las Orientaciones del MINSAL (2010) definen comunidad sólo en base a territorio compartido por un grupo de individuos.

Por otra parte, la OMS propone medidas para trabajar en todas las áreas del desarrollo y con todo tipo de discapacidades, lo que tiene como finalidad la inclusión de todas las personas con discapacidad en la sociedad. Mientras que la propuesta del MINSAL apunta sólo a la rehabilitación de discapacidades físicas, excluyendo las sensoriales y psíquicas. Asimismo por ser un documento desarrollado por el ministerio de salud, describe acciones que abordan solamente las intervenciones en salud y el acceso a éstas, dejando fuera los demás sectores.

En las Guías para RBC se establece que las estrategias deben surgir desde la comunidad misma y que para esto se deben desarrollar procesos de sensibilización y educación previos que permitan a las personas con discapacidad y su comunidad identificar sus problemáticas y trabajar para su resolución. Mientras que las orientaciones metodológicas proponen una estrategia que surge desde niveles centrales, donde los profesionales están encargados de desarrollar diagnósticos y planes de intervención. Si bien se incluye a la comunidad en el proceso, es el profesional el encargado de dirigir.

Finalmente la OMS establece la capacitación de miembros de la comunidad para lograr intervenir y trabajar con los propios involucrados como una de las principales estrategias para el desarrollo de programas de RBC. Mientras que el MINSAL pareciera proponer una extensión de servicios profesionales en la comunidad, donde las atenciones son esencialmente entregados dentro de ella, pero no existe una participación o involucramiento de los individuos en el proceso de rehabilitación. Por lo tanto este modelo de acercamiento no se consideraría basado en la comunidad.

Si bien en Chile se define la implementación de una estrategia de RBC en los servicios de APS, pareciera que los conceptos que se adoptan se asemejan a los primeros documentos publicados por la OMS y se distancian bastante de los conocimientos y conceptos actuales de la RBC a nivel internacional.

\section{Resistencias nacionales a la implementación de la estrategia de RBC}

De acuerdo a lo descrito anteriormente, es posible apreciar que Chile se encuentra en los primeros años de desarrollo de la RBC, donde se 
experimenta recién la transición de un modelo biomédico a uno biopsicosocial. Así las políticas de salud apuntan hacia una lógica inclusiva utilizando la estrategia de RBC como uno de los mecanismos para lograrlo.

A pesar de que existen intenciones declaradas a través de documentos y bajo las propias necesidades de la comunidad, es posible detectar ciertas barreras que dificultan y/o retrasan la implementación de la RBC en las atenciones de salud actuales en el país. Frente a estas características, ¿es posible alcanzar las metas y objetivos propuestos para la RBC a nivel nacional?

Las barreras nacionales descritas a continuación serán consideradas como resistencias, pues sus resultados o acciones apuntan en sentido contrario a lo planteado por el MINSAL para lograr implementar la RBC en la APS en Chile.

a) Resistencia social: Basada en lo expuesto por Instituto Nacional de Rehabilitación Pedro Aguirre Cerda (INRPAC) en 2005, en relación a las características sociales que retrasan el proceso de implementación de la RBC, se encuentran el desconocimiento de la estrategia por parte de la sociedad, pues no se ha realizado un proceso de difusión de la nueva forma de rehabilitación por lo que la comunidad no conoce la real importancia ni el peso de sus actos dentro del nuevo modelo y estrategia de salud. Además se aprecia un escaso empoderamiento de las personas con discapacidad para que logren asumir un rol activo y participativo en su comunidad, debido principalmente a un desconocimiento de sus derechos y deberes. Asimismo existe una insuficiente sensibilización, que podría interferir en el proceso de reconocimiento de las capacidades y derechos de las personas con discapacidad, manteniendo las prácticas de discriminación y exclusión hacia este grupo.

b) Resistencias política e institucional: De acuerdo a un análisis e investigación realizados es posible corroborar que los compromisos que adquirió el MINSAL en 2004 aún no son cumplidos en su totalidad, pues no existe en la actualidad una Política Nacional de Rehabilitación y Discapacidad, lo que podría estar interfiriendo en la puesta en práctica de la RBC e impidiendo el acceso de todas las personas con discapacidad a las intervenciones de salud. Asimismo, el desarrollo de un sistema de rehabilitación física y sensorial con base en la comunidad, aún no es implementado con éxito. Si bien en los CCR se apunta a los servicios de rehabilitación física, la discapacidad sensorial todavía no se encuentra dentro de los programas. Además no existen en la actualidad manuales chilenos que clarifiquen y entreguen estrategias para que en los servicios de APS se implemente la RBC.

Por otra parte se carece de investigaciones que validen la RBC, tanto desde la percepción de la propia comunidad como en aspectos de costo efectividad de la nueva estrategia. Además, a pesar de la amplia oferta de CCR que existen en la actualidad, existen solamente 4 documentos $^{4}$ donde se difunden las experiencias exitosas que han tenido estos centros a lo largo de los años1. Todo lo anterior podría llevar a que los equipos profesionales desconfíen de estas estrategias novedosas o finalmente pierdan la confianza.

c) Resistencia profesional : En cuanto a las resistencias profesionales, si se observan detenidamente las mallas curriculares propuestas por la Universidad de Chile y expuestas en su página web, suponiendo que al ser una universidad estatal debiese replicar las nuevas bases del conocimiento vinculado al beneficio y bienestar de la población, se esperaría encontrar que dentro de los contenidos tratados durante la formación profesional de los estudiantes de

4 Documentos a cargo del MINSAL que describen la puesta en práctica de proyectos de RBC en CCR: "Sistematización de la experiencia de Rehabilitación Basada en la Comunidad en Instituto Nacional de Rehabilitación Pedro Aguirre Cerda" (2005), "Sistematización de la experiencia de Rehabilitación Basada en la Comunidad en Monte Patria" (2008), "Sistematización de la Experiencia de Rehabilitación Basada en la Comunidad en Chimbarongo" (2010) y "Sistematización de la experiencia de Rehabilitación Basada en la Comunidad en la Reina" (2012). 
las carreras de Terapia Ocupacional y Kinesiología, existiera un gran porcentaje de ramos vinculados a la RBC y trabajo en comunidad, debido a que son los miembros fundamentales de los equipos de rehabilitación propuestos por el MINSAL en 2010. Sin embargo, parece bastante sorprendente que los contenidos establecidos para la formación de kinesiólogos se refieran principalmente a aspectos biomédicos. Por su parte, la Escuela de Terapia Ocupacional se encuentra actualmente en la puesta en marcha de un proyecto de innovación curricular, que se caracteriza por entregar a sus estudiantes contenidos vinculados a la nueva realidad de la población y de la salud en Chile. A pesar de lo anteriormente planteado, es importante notar que las prácticas, tanto previas como profesionales, están principalmente vinculadas a aspectos clínicos, lo que podría provocar un distanciamiento de los futuros profesionales de la salud con la RBC.

Por otra parte, en relación a la formación de post grado ofrecida por la Universidad de Chile, no existen planes que permitan a los estudiantes egresados continuar su formación en el ámbito comunitario, lo que podría dificultar aún más su preparación para hacer frente a las necesidades y a trabajar con las acciones propuestas por la nueva estrategia. No obstante, es importante considerar que a pesar de la escasa disponibilidad académica relacionada con la RBC a nivel estatal, sí existen propuestas de instituciones privadas ${ }^{5}$.

Con el precedente de las barreras sociales, políticas, institucionales y profesionales anteriormente planteadas e intentando dar respuesta a la pregunta: ¿Es posible alcanzar las metas y objetivos propuestos para la RBC a nivel nacional?, se podría decir que a pesar de los esfuerzos que apuntan a implementar la RBC en el país, todavía hay necesidades insatisfechas, derechos vulnerados y muy pocas personas con discapacidad que participen

5 En referencia a disponibilidad de cursos de postgrado en la Universidad Andrés Bello. como ciudadanía activa. Por esto, se podría considerar que aún existe en Chile una realidad alejada de la valorización de la comunidad, donde las personas con discapacidad todavía se encuentran en el proceso de asumir su responsabilidad social y exigir el cumplimiento real de sus derechos. De igual manera, la comunidad pareciera estar lejos de validar y promover el cumplimiento y respeto de los derechos de las personas con discapacidad bajo la lógica inclusiva, por lo que la real implementación de un plan de RBC pareciera encontrarse muy lejano. Sumado a lo anterior, si las instancias formales de salud y los organismos de gobierno no están capacitados de forma adecuada y no se asume e implementa desde niveles centrales planes para favorecer las condiciones sociales que permitan el desarrollo de la nueva forma de rehabilitación, la lógica biomédica y asistencialista podría seguir rigiendo las prácticas de salud y de todos los sectores, alejándose de los objetivos y metas planteados por la RBC.

\section{COnClusiones}

Producto del desarrollo, historicidad y progresión de la RBC tanto a nivel nacional e internacional, es posible considerar a esta estrategia como una idea novedosa y vanguardista en la forma de mirar y valorizar a las personas con discapacidad, las comunidades y los recursos sociales.

Chile no ha quedado ajeno a las estrategias propuestas internacionalmente. Mediante esfuerzos y políticas, ha intentado posicionar la nueva forma de rehabilitación como objetivo de intervención en salud. Sin embargo, la realidad nacional actual ha puesto barreras para su implementación, debido a que al parecer no cuenta con un soporte socio-político necesario para el cambio.

Basada en el Informe Mundial sobre la Discapacidad publicado por la OMS en 2011, donde se planteó una lista de obstáculos que podrían interferir en la implementación de los programas de RBC, aquellos que se relacionan con la realidad chilena son los siguientes: 
Carencia de políticas y normas que incluyan a las personas con discapacidad: pues si bien existe actualmente la Ley 20.422 y el MINSAL ha entregado orientaciones que apuntan a la inclusión de las personas con discapacidad en el sector salud, aún faltan políticas que apunten a una real inclusión de este grupo hacia los demás sectores del desarrollo.

Actitudes negativas de la sociedad frente a las personas con discapacidad: ya que se carece de un proceso masivo de sensibilización de la comunidad por lo que frente al desconocimiento de las capacidades y potencialidades de las personas con discapacidad, socialmente aún existen conductas de discriminación.

Escasa cobertura de servicios y accesibilidad a ellos: no sólo del sector salud, sino que también de accesibilidad espacios públicos, a instancias formales de trabajo y educación.

Desactualizada información disponible en relación a estadísticas y datos de discapacidad en el país: si se considera que la información estadística específica de la discapacidad data del año 2004 y no existen actualizaciones del tema, es posible decir que actualmente se desconoce de la realidad en la que se desenvuelve a diario este grupo.

Frente a este desfavorable panorama relacionado con las características socioculturales chilenas, se vuelve imperioso adaptar a la realidad actual del país las guías propuestas por la OMS en 2012, con la finalidad de crear un patrón que facilite la implementación progresiva de la RBC. Asimismo, se debe sumar al proceso la difusión de la información tanto a nivel central como en las distintas instancias de intervención para hacer público los conocimientos y facilitar la utilización y socialización de ellos. De la misma manera, para evitar una resistencia por parte de los profesionales de los equipos de salud, podría favorecer el trabajo conjunto desde la formación, utilizando ideas como la innovación de mallas curriculares e inclusión de prácticas profesionales relacionadas a lo comunitario. Sumado a esto, parece fundamental que las casas de estudio estatales posean dentro de su formación de postgrado programas vinculados a la RBC, acercando desde los conocimientos a los futuros profesionales encargados de implementar los cambios.

Por otra parte, considerando lo expuesto por Kronenberg, Pollard y Simó (2007), para lograr incluir a la comunidad dentro del nuevo concepto de rehabilitación, es fundamental que exista una actitud positiva hacia las personas con discapacidad. Esto podría lograrse mediante campañas publicitarias y educación social en relación a capacidades, potencialidades y derechos de estas personas.

Debido a que un "país no puede avanzar de forma sostenida sin reconocer los principios de derechos humanos" (Oficina del Alto Comisionado para los Derechos Humanos, 2006), es hora de que en Chile exista prioridad en relación a las personas con discapacidad y el respeto de sus derechos desde todos los aspectos que llevan a la inclusión y por parte de todos los actores de la comunidad, incluyendo a los propios afectados. Para lo anterior, los terapeutas ocupacionales juegan un rol fundamental, pues se encuentran capacitados para promover la inclusión y participación social de las personas con discapacidad y trabajar dentro de su contexto, promoviendo su empoderamiento y facilitando su acceso a los distintos sectores. Si bien la Terapia Ocupacional tiene muchas potencialidades en el campo de la RBC, también existen muchas deudas que saldar con la comunidad, comenzando por la actualización de los conocimientos.

\section{BibLIOGRAFÍA}

Alcalde, J. (1981). Las relaciones económicas internacionales en la década del 80. Recuperado el 16 de Febrero de 2013, de Estudios Internacionales: Revista del Instituto de Estudios Internacionales: http://www.revistaei.uchile.cl/index.php/REl/article/ viewFile/16279/21393

Escuela de Kinesiología de la Universidad de Chile. (s.f.). Malla Curricular Escuela de Kinesiología. Recuperado el 22 de Febrero de 2013, de http://kinesiologia.med.uchile.cl/images/malla.pdf

Escuela de Postgrado de la Universidad de Chile. (s.f.). Programas de Especialistas. Recuperado el 22 de Febrero de 2013, de http:// www.postgradomedicina.uchile.cl/ 
Escuela de Terapia Ocupacional de la Universidad de Chile. (s.f.). Malla Curricular Escuela de Terapia Ocupacional. Recuperado el 22 de Febrero de 2013, de http://tocupacional.med.uchile.cl/ images/malla.pdf

Fondo Nacional de la Discapacidad. (2006). Discapacidad y Rehabilitación. Capítulo 2. En Discapacidad en Chile: pasos hacia un modelo integral del funcionamiento humano (págs. 20 - 40). Santiago.

Fondo Nacional de la Discapacidad. (2004). ENDISC-CIF Primer Estudio Nacional de la Discapacidad en Chile. Santiago.

Freire, P. (1970). Pedagogía del Oprimido. Recuperado el 3 de Marzo de 2013, de http://www.ensayistas.org/critica/liberacion/varios/ freire.pdf

Infante, A., \& Paraje, G. (2010). La Reforma de Salud en Chile. Recuperado el 3 de Marzo de 2013, de Programa de las Naciones Unidas para el Desarrollo: http://www.pnud.cl/areas/ReduccionPobreza/2012/2010_5.pdf

Instituto Nacional de Rehabilitación Pedro Aguirre Cerda. (2010). Guías de Cuidados Básicos de Rehabilitación. RBC. En C. García, Estrategia de RBC (págs. 23-27). Santiago.

Instituto Nacional de Rehabilitación Pedro Aguirre Cerda. (2010). Guías de Cuidados Básicos de Rehabilitación. RBC. En A. Vargas, Modelos en Discapacidad (págs. 15-22). Santiago

Instituto Nacional de Rehabilitación Pedro Aguirre Cerda. (2010). Guías de Cuidados Básicos en Rehabilitación. RBC. En C. García, ¿Qué es gestión comunitaria? (págs. 28-29). Santiago.

Instituto Nacional de Rehabilitación Pedro Aguirre Cerda. (2010). Guías de Cuidados Básicos en Rehabilitación. RBC. En M. S. Astorga, Redes Sociales (págs. 30-32). Santiago.

Instituto Nacional de Rehabilitación Pedro Aguirre Cerda. (2005). Sistematización Rehabilitación con Base Comunitaria INRPAC. Santiago.

Kolmans, E. (s.f.). La educación popular, los enfoques educativos modernos y la metodología CAC. Recuperado el 22 de Marzo de 2013, de http://www.pidaassa.org/mvertical/meto_herram/ La_educacion_popular_y_CaC.pdf

Krause Jacob, M. (2001). Hacia una redefinición del concepto de comunidad. Recuperado el 3 de Febrero de 2013, de Revista de Psicología de la Universidad de Chile: http://www.revistapsicologia.uchile.cl/index.php/RDP/article/viewFile/18572/19618

Kronenberg, F., Simó, S., \& Pollard, N. (2007). Terapia Ocupacional sin Fronteras: Aprendiendo del espíritu de supervivientes. En H. Fransen, Los desafíos de la Terapia Ocupacional en la Rehabilitación basada en la Comunidad (págs. 168-184). Madrid: Médica Panamericana.

Ministerio de Salud del Gobierno de Chile. (2010). Orientaciones Metodológicas para el Desarrollo de las Estrategias de Rehabilitación en APS 2010-2014. Recuperado el 10 de Febrero de 2013, de SENADIS: http://www.senadis.gob.cl/descargas/centro/tematicos/salud/ORIENTACIONES-METODOLOGICAS-REHABILITACION.pdf

Ministerio de Planificación del Gobierno de Chile. (2010). Ley No 20.422. Recuperado el 15 de Febrero de 2013, de http://www. leychile.cl/Navegar?idLey $=20422$

Ministerio de Planificación del Gobierno de Chile. (1994). Ley de Integración Social de las Personas con Discapacidad No 19.284.
Recuperado el 10 de Febrero de 2013, de http://www.mineduc. cl/biblio/documento/200810271405400.Ley_de_Integracion_ Social_de_las_Personas_con_Discapacidad-19.284.pdf

Oficina del Alto Comisionado de las Naciones Unidas para los Derechos Humanos. (2006). Preguntas frecuentes sobre el Enfoque de Derechos Humanos en la Cooperación para el Desarrollo. Recuperado el 15 de Febrero de 2013, de Oficina del Alto Comisionado para los Derechos Humanos: http://www.ohchr.org/ Documents/Publications/FAQsp.pdf

Organización de las Naciones Unidas. (2006). Convención sobre los Derechos de las Personas con Discapacidad y Protocolo Facultativo. Recuperado el 7 de Febrero de 2013, de Servicio Nacional de la Discapacidad: http://www.senadis.gob.cl/descargas/centro/ internacionales/Convencion-Internacional.pdf

Organización de las Naciones Unidas. (1993). Normas Uniformes sobre la igualdad de oportunidades para las personas con discapacidad. Recuperado el 2 de Febrero de 2013, de http://www. un.org/spanish/disabilities/default.asp?id=498

Organización Internacional del Trabajo; Organización de las Naciones Unidas para la Educación, la Ciencia y la Cultura \& Organización Mundial de la Salud. (2004). RBC Estrategia para la rehabilitación, la igualdad de oportunidades, la reducción de la pobreza y la integración social de las personas con discapacidad. Documento de Posición Conjunta. Recuperado el 4 de Febrero de 2013, de Organización Mundial de la Salud: http://whqlibdoc.who.int/publications/2005/9243592386_spa.pdf

Organización Internacional del Trabajo; Organización de las Naciones Unidas para la Educación, la Ciencia y la Cultura \& Organización Mundial de la Salud. (1994). Rehabilitación Basada en la Comunidad con y para Personas con Discapacidad. Ponencia Conjunta. Recuperado el 7 de Febrero de 2013, de UNESCO: http://unesdoc.unesco.org/images/0011/001117/111772so.pdf

Organización Mundial de la Salud. (2012). Rehabilitación Basada en la Comunidad. Guías para la RBC. En Folleto Introducción. Ginebra.

Organización Mundial de la Salud. (2012). Rehabilitación Basada en la Comunidad. Guías para la RBC. En Componente de Salud. Ginebra.

Organización Mundial de la Salud. (2012). Rehabilitación Basada en la Comunidad. Guías para la RBC. En Componente de Educación. Ginebra.

Organización Mundial de la Salud. (2012). Rehabilitación Basada en la Comunidad. Guías para la RBC. En Componente de Subsistencia. Ginebra.

Organización Mundial de la Salud. (2012). Rehabilitación Basada en la Comunidad. Guías para la RBC. En Componente Social. Ginebra.

Organización Mundial de la Salud. (2012). Rehabilitación Basada en la Comunidad. Guías para la RBC. En Componente de Fortalecimiento. Ginebra.

Organización Mundial de la Salud. (2011). Resumen Informe Mundial sobre la Discapacidad. Recuperado el 12 de Febrero de 2013, de Organización Mundial de la Salud: http://www.who. int/disabilities/world_report/2011/summary_es.pdf

Organización Mundial de la Salud. (1978). Alma Ata 1978. Atención Primaria de Salud. Recuperado el 2 de Febrero de 2013, 
de Organización Mundial de la Salud: http://whqlibdoc.who.int/ publications/9243541358.pdf

Organización Panamericana de la Salud. (2011). Implementación del modelo biopsicosocial para la atención de las personas con discapacidad a nivel nacional. Recuperado el 7 de Mayo de 2013, de http://new.paho.org/els/index2.php?option=com_ docman\&task=doc_view\&gid=870\&ltemid=99999999
Oyarzún, E. (2008). Modelo biomédico y Modelo biopsicosocial. Recuperado el 12 de Febrero de 2013, de https://www.ucursos.cl/medicina/2008/2/MPRINT25/1/material_docente/ previsualizar?id_material $=182943$

Universidad Andrés Bello. (s.f.). Postgrados. Recuperado el 22 de Febrero de 2013, de http://www.postgradounab.cl/ 